\title{
La limitation des droits économiques, sociaux et culturels en RDC pendant la période de l'état d'urgence sanitaire : état des lieux, défis et perspectives
}

\author{
Par William KATEMBO KASILAM*
}

\begin{abstract}
Résumé
Des circonstances exceptionnelles, extrêmes, imprévisibles, peuvent amener un État, sur un territoire donné, pour une durée déterminée, à une restriction des droits de l'homme pendant la période de l'état d'urgence. Certaines libertés fondamentales peuvent être restreintes pendant cette période. En RDC, l'ordonnance du Président de la République du 24 mars 2020 qui a proclamé l'état d'urgence sanitaire pour la lutte contre la propagation de la pandémie de Covid-19 a limité un grand nombre des droits de l'homme. Cet article pose la question de savoir si tous les droits de l'homme peuvent être limités dans pareil circonstance et dans le cas d'une réponse négative, quels sont les droits qui peuvent être limités et quelles sont les exigences de cette limitation sur le plan temporaire et spatial.
\end{abstract}

\begin{abstract}
Exceptional, extreme, unforeseeable circumstances may lead a State, within a given territory, for a given period of time, to a restriction of human rights during a state of emergency. Certain fundamental freedoms may be restricted during this period. In the DRC, the Presidential Order of March 24, 2020, which declared a state of health emergency to combat the spread of the Covid-19 pandemic, limited a large number of human rights. This article raises the question of whether all human rights can be limited in such circumstances and, if not, which rights can be limited and what are the requirements of this limitation on a temporary and spatial level.
\end{abstract}

\section{Introduction}

Assigner une population à domicile, fermer les établissements scolaires, les universités, les restaurants, les cafés, limiter les sorties de loisir et restreindre drastiquement la circulation des personnes aux frontières extérieures du territoire national comme en son sein dans un contexte de pandémie de covid-19, l'état de « l'Etat de droit » prôné par le Chef de l'Etat a brusquement basculé en RDC. Pour nombre de destinataires de ces mesures une telle situation est inédite à propos de ce confinement domiciliaire durable imposée à une nation puisque même dans le ghetto de Varsovie, il était possible de circuler malgré que ce ghetto

* Licencié en droit de l’Université de Kinshasa, E-mail : williamkatembo85@gmail.com. 
fût l'antichambre de la mort alors que le confinement du début de l'année 2020 visait à protéger la vie.

L'état d'urgence est une mesure prise par un gouvernement en cas de péril imminent dans un pays. Certaines libertés fondamentales peuvent être restreintes. Des circonstances exceptionnelles, extrêmes, imprévisibles, peuvent amener un État, sur un territoire donné, pour une durée déterminée, à une restriction des lois, réputées démocratiques, et jugées insuffisantes pour faire face à un danger public en cours ou imminent, contrairement aux normes de l'État de droit courant. Ces mesures sont souvent à l'origine de l'état d'urgence.

Les droits socio-économiques sont des droits humains concernant les conditions sociales et économiques essentielles à une vie digne et libre, et qui sont liés au travail, à la sécurité sociale, à la santé, à l'éducation, à l'alimentation, à l'eau, au logement, à un environnement sain et voire même à la culture. Il s'agit ici, d'une catégorie des droits humains qui constituent un cadre commun de valeurs et de normes universellement reconnues et établissent l'obligation qui revient aux États d'agir d'une certaine manière ou de renoncer à certains actes. Il s'agit d'un instrument important pour amener les États, et de plus en plus les acteurs non étatiques, à répondre des violations des droits ainsi que pour mobiliser les efforts collectifs visant le développement de communautés et de structures mondiales favorables à la justice économique, au bien-être sociale, à la participation et à l'égalité. Les droits humains sont universels, inaliénables, interdépendants et indivisibles.

Nous devons avoir à l'esprit que l'Etat moderne doit protéger les droits de tous. Il est régi par son droit interne et est également soumis au droit international en tant que membre de la communauté des nations. L'Etat moderne est d'abord et avant tout un Etat au service de tous. C'est un Etat responsable et exigeant à la fois vis-à-vis de lui-même et des services publics qu'il doit rendre des comptes à la population que vis-à-vis des gouvernés. Il doit veiller au bien-être de sa population. Il doit s'assurer que les citoyens jouissent aussi bien des droits civils et politiques que des droits socioéconomiques ${ }^{1}$

En RDC, lorsque des circonstances graves menacent, d'une manière immédiate, l'indépendance ou l'intégrité du territoire national ou qu'elles provoquent l'interruption du fonctionnement régulier des institutions, le Président de la République proclame l'état d'urgence ou l'état de siège après concertation avec le Premier ministre et les Présidents des deux Chambres du Parlement ${ }^{2}$. Mais, en dépit de cette restriction des droits de l'homme, il y a des droits qui ne peuvent pas être affectés par cette situation exceptionnelle. C'est ainsi qu'en aucun cas, et même lorsque l'état de siège ou l'état d'urgence aura été proclamé, il ne peut être dérogé aux droits et principes fondamentaux énumérés ci-après : le droit à la vie; l'interdiction de la torture et des peines ou traitements cruels, inhumains ou dégradants; l'interdiction de l'esclavage et de la servitude; le principe de la légalité des infractions et

1 André MBATA BETUKUMESU MANGU, Abolition de la peine de mort et constitutionnalisme en Afrique, Paris, 2011, p. 144.

2 Article 85 de la Constitution de la RDC du 18 février 2006, in J.O.R.D.C, 47ème année, numéro spécial du 18 février 2006 telle que modifiée en 2011. 
des peines; les droits de la défense et le droit de recours; l'interdiction de l'emprisonnement pour dettes; la liberté de pensée, de conscience et de religion ${ }^{3}$.

Au niveau international, il est prévu que dans le cas où un danger public exceptionnel menace l'existence de la nation et est proclamé par un acte officiel, les États parties au Pacte international relatif aux droits civils et politiques de l'ONU peuvent prendre, dans la stricte mesure où la situation l'exige, des mesures dérogeant aux obligations prévues dans le présent Pacte, sous réserve que ces mesures ne soient pas incompatibles avec les autres obligations que leur impose le droit international et qu'elles n'entraînent pas une discrimination fondée uniquement sur la race, la couleur, le sexe, la langue, la religion ou l'origine sociale. (...). La proclamation de l'état d'urgence ne permet pas de déroger à certains droits fondamentaux et interdictions absolues, dont en particulier le « droit à la vie », l'interdiction de la torture et des traitements inhumains et dégradants, l'esclavage, la servitude, la loi rétroactive et la « reconnaissance de la personnalité juridique » la « liberté de penser, de conscience et de religion ».

Cet article envisage de faire une analyse textuelle contextualisée à la période de la durée de l'Etat d'urgence sanitaire en RDC en ce qui concerne les droits de l'homme dont la jouissance ou l'exercice à été limité avec un regard particulier sur les droits économiques, sociaux et culturels partant des principaux textes juridiques en vigueur confrontés aux mesures ayant régis la période de l'état d'urgence sanitaire en RDC.

\section{A. Les droits de l'homme potentiellement limitables par l'Etat d'urgence}

Il est impérieux d'examiner le fondement de la limitation des droits de l'homme tout en répondant à la question de savoir si c'est tous les droits de l'homme qui peuvent faire l'objet de limitation. Ainsi, il serait question de voir le fondement idéologique de la limitation des droits de l'homme par la suite, voir quels sont les droits de l'homme limitable en RDC et le fondement juridique y afférent.

\section{Fondement idéologique de limitation des droits de l'homme}

Les limitations des droits de l'homme durant la période de la pandémie de Covid-19 sont généralisées et au risque de devenir, ainsi, pour un temps, la norme. Tel est la plus grande inquiétude naissante suite aux mesures de l'Etat d'urgence proclamé par les dirigeants du pays. Tout de même, les limitations sont inhérentes aux droits de l'homme; il ne s'agit pas d'opposer les premières aux seconds : «La préservation de l'intérêt général et des intérêts d'autrui n'est pas l'« Autre » des droits et libertés, mais, dans une très large mesure, une condition de leur effectivité. Tel est le contexte général qui guide notre appréhension de la situation.

3 Article 61 de la Constitution de la RDC, note 2. 
L'Homme tel que conçu dans l'univers des droits de l'homme est pensé de manière autonome : il n'est pas seulement la partie d'un tout qui le transcenderait; ses droits lui permettent de s'autodéterminer. Pour autant, il n'est pas placé hors du groupe, comme détaché de la cité; il est, au contraire, " projeté dans une relation avec son environnement constitué par autrui, par la société, par le Pouvoir». Seule la communauté permet, d'ailleurs, « le libre et plein développement de sa personnalité »; c'est pourquoi, il a des devoirs envers elle. La crise sanitaire actuelle met en lumière cette dimension des droits de l'homme qui, en période «normale », se manifeste davantage par touches impressionnistes. Elle rappelle que ces droits reposent sur une « tentative permanente de combinaison des tendances antagonistes à la revendication des droits et à l'organisation des droits, des besoins simultanés et contraires de liberté et de Pouvoir ». L'État peut ou doit, dans certains cas, limiter les libertés pour faire respecter les droits d'autrui et « satisfaire aux justes exigences de la morale, de l'ordre public et du bien-être général dans une société démocratique ». C'est dans cet esprit que les ordonnances du Président de la République sur l'état d'urgence et les mesures d'applications y relatives peuvent être comprises.

Reste que si des limitations des droits sont possibles, il existe aussi des limites à ces limitations. Lorsque l'État entend déroger aux droits de l'homme, il doit, conformément aux règles internationales, satisfaire à certaines conditions formelles et procédurales. Il doit s'assurer, aussi, que les mesures adoptées n'entraînent pas une « discrimination fondée uniquement sur la race, la couleur, le sexe, la langue, la religion ou l'origine sociale ». Pour le reste, les limitations sont principalement affaire de nécessité et de proportionnalité : la mesure en cause doit contribuer à la réalisation du but poursuivi qui doit être légitime (en l'occurrence protéger la santé et la vie) et les inconvénients causés ne doivent pas être démesurés par rapport à ce but; il ne doit pas exister, non plus, d'autre mesure appropriée qui serait moins coûteuse en termes de libertés. Les limitations de droits s'apprécient ainsi à l'aune des circonstances; elles ne se laissent pas enfermer dans un modèle donné d'avance. L'empirisme sur lequel elles reposent peut expliquer que les titulaires de ces droits rencontrent parfois des difficultés à déterminer si une situation déterminée constitue ou non une violation.

\section{Fondement juridique de la limitation des droits de l'homme en RDC}

Le régime juridique applicable à la limitation des droits de l'homme en RDC comprend deux grands ensembles. Le premier, comprend toutes les normes nationales relatives à la promotion, la protection et la défense des droits de l'homme, mais également leur limitation. Le second quant à lui est constitué des normes du droit international en vigueur en RDC.

Au niveau national, les circonstances et les mécanismes pour limitation des droits de l'homme en RDC apparaissent de prime à bord dans la Constitution en vigueur. Il y est prévu qu'en aucun cas, et même lorsque l'état de siège ou l'état d'urgence aura été proclamé conformément aux articles 85 et 86 de la présente Constitution, il ne peut être dérogé aux 
droits et principes fondamentaux suivant : le droit à la vie; l'interdiction de la torture et des peines ou traitements cruels, inhumains ou dégradants; l'interdiction de l'esclavage et de la servitude; le principe de la légalité des infractions et des peines; les droits de la défense et le droit de recours; l'interdiction de l'emprisonnement pour dettes; la liberté de pensée, de conscience et de religion ${ }^{4}$.

Les dérogations aux droits de l'homme protégés au niveau international ne peuvent intervenir qu' « en cas de danger public exceptionnel », lorsque l'existence même de la nation est en cause et « dans la stricte mesure où la situation l'exige $»^{5}$. Ce danger exceptionnel peut être lié à un conflit armé, une catastrophe, une menace terroriste ou une pandémie en termes de guerre sanitaire, mais quoi qu'il en soit, la situation doit être particulièrement grave. Deux conditions doivent être réunies : d'une part, la menace doit être dirigée, à la fois, contre la totalité de la population et contre tout ou partie du territoire; d'autre part, la situation doit menacer l'indépendance politique de l'État, son intégrité territoriale, les fonctions régulier de ses institutions ou, comme dans le cas d'une crise sanitaire, l'intégrité physique de sa population.

Si ces conditions doivent être réunies, l'État dispose toutefois d'une grande latitude dans leur appréciation : c'est lui qui décide en premier lieu et sans étape préalable d'user de son droit de déroger. Il est considéré comme étant le plus à même pour déterminer si un danger public existe; le contrôle international sur l'opportunité de cette décision est faible, pour ne pas dire inexistant. Dans ce type d'hypothèse, l'impératif d'efficacité prend, vraisemblablement, le pas sur l'impératif de légalité ${ }^{6}$.

En revanche, l'État doit pouvoir justifier chaque mesure concrète qui découle de cette situation. C'est à ce stade que le contrôle des organes internationaux des droits de l'homme se fait plus marqué. L'approche de ces organes est conséquentialiste en ce qu'elle se focalise principalement sur les effets de la décision ${ }^{7}$. Il ne s'agit pas tant de juger les motifs qui ont conduit l'État à déclarer l'état d'urgence que d'apprécier les conséquences concrètes de cette décision sur les droits de l'homme.

Pour être conforme aux exigences internationales, toute mesure doit être nécessaire, c'est-à-dire « dirigée contre un danger réel, manifeste, présent ou imminent et ne peut être imposée par simple crainte d'un danger potentiel ». Elle doit être, aussi, strictement proportionnée, c'est-à-dire que l'intensité de l'atteinte aux droits et libertés doit être fonction de la gravité de la menace ${ }^{8}$. Avant de l'adopter, les autorités doivent s'assurer que les autres moyens, moins liberticides, sont manifestement inefficaces. Ainsi, si le port de masques ou des dépistages massifs sont susceptibles de constituer une réponse appropriée à la pandé-

4 Article 61 de la Constitution de la RDC.

5 Article 4 du Pacte international relatif aux droits économiques, sociaux et culturels ( PIDESC ») du 16 décembre 1966.

6 LUDOVIC HENNEBEL ET HELENE TIGROUDJA, Traité de droit international des droits de l'homme, Paris, Pedone, 2016, p. 29.

7 WACHSMANN, Les droits de l'homme, Paris, 2002, p.5.

8 Haut-Commissariat des Nations Unies aux droits de l'homme, « Emergency measures and covid-19. 
mie, ils doivent être préférés aux autres mesures, en particulier aux interdictions de sortie, ce qui pose question en cas de pénurie de ce type de matériel sanitaire.

Dans les situations d'état d'urgence, déroger à la liberté de mouvement et de réunion est, en général, suffisant selon le Comité des droits de l'homme. S'agissant de la crise du covid-19, toutefois, de nombreux autres droits ont été impactés, en particulier les droits économiques, sociaux et culturels notamment, s'agissant du droit au travail, il a été soit aménagé (avec le télétravail) en dépit des infrastructures adéquates quasi inexistantes, soit entravé, selon les cas.

Certains droits ne peuvent souffrir d'aucune limitation, y compris en cas de danger exceptionnel; les organes internationaux sont particulièrement vigilants sur ce point. La liste de ces droits indérogeables varie quelque peu d'une convention de droits de l'homme à une autre, mais il existe un noyau dur de droits absolus. Celui-ci - qui interdit notamment la torture ou les traitements inhumains et dégradants ${ }^{9}$ ainsi que les atteintes arbitraires à la vie - vise à protéger l'irréductible humain ${ }^{10}$. À ce titre, il est interdit, en particulier en cas d'épidémie, de soumettre une personne, sans son libre consentement, à une expérience médicale ou scientifique ${ }^{11}$ : ni la liberté de recherche ${ }^{12}$, ni les préoccupations de santé publique ne justifient qu'un médicament soit testé sur une personne qui n'a pas donné son accord dans le seul but d'en vérifier les propriétés.

Par ailleurs, l'État qui décide de déroger aux droits de l'homme protégés au niveau international est tenu de respecter certaines règles formelles. Il doit adopter un " acte officiel » proclamant le danger public exceptionnel de manière à en informer sa population. Il doit aussi le notifier au Secrétaire général de l'Organisation des Nations Unies, s'agissant du PIDCP, et signaler ainsi la situation aux autres États parties au traité. A ce niveau l'intérêt que l'État qui n'a pas activé la clause de dérogation ne peut arguer devant l'organe international de droits de l'homme de la situation d'urgence pour justifier d'éventuelles violations de droits individuels en cas de " plainte $»^{13}$. Dans sa notification, l'État fournit, en principe, des explications précises et circonstanciées sur les motifs qui l'ont conduit à prendre de telles mesures et sur les libertés ou droits auxquels il entend déroger. Espérons que l'Etat congolais a pu s'acquitter de cette obligation internationale du moins, pas négligeable.

9 Article 4, par. 2 du PIDCP et article 15, par. 2 de la CEDH.

10 MIREILLE DELMAS-MARTY, « Le crime contre l'humanité, les droits de l'homme et l'irréductible humain », Revue de science criminelle et de droit pénal comparé, 1994, n 3, p. 477- 490.

11 Art. 7 du PIDCP.

12 Art. 15, par. 3 du PIDESC.

13 LUDOVIC HENNEBEL et HELENE TIGROUDJA, Traité de droit international des droits de l'homme, Paris, Pedone, 2016, p. 73. 


\section{B. Les droits socio-économiques limités par l'Etat d'urgence sanitaire en RDC}

\section{L'Analyse du contenu de son cadre juridique}

L'état d'urgence sanitaire a été proclamé en RDC par l'Ordonnance numéro 20/014 du 24 mars 2020 portant proclamation de l'état d'urgence sanitaire pour faire face à la pandémie de Covid-19. Cette Ordonnance a été suivi de plusieurs mesures d'application notamment l'Arrêté ministériel No. 25/CAB/VPM/ MININTERSECAC/GKM/06/2020 du 25 mars 2020 portant modalités pratiques d'exécution des mesures de limitation de la propagation de la pandémie de Covid-19; Ordonnance n²0-018 du 6 avril 2020 portant création, organisation et fonctionnement d'un Fonds national de solidarité contre le Coronavirus, en sigle « FNSCC; Ordonnance n ${ }^{\circ} 20 / 033$ du 02 mai 2020 modifiant et complétant l'ordonnance n `20/018 du 06 avril 2020 portant création, organisation et fonctionnement d'un Fonds national de solidarité contre le Coronavirus, en sigle « FNSCC; Ordonnance n²0/029 du 23 avril 2020 portant mesures complémentaires nécessaires pour faire face à l'épidémie au Covid 19; voire même l'Ordonnance n²0/036 du 02 mai 2020 portant création, organisation et fonctionnement d'une commission ad hoc dénommée « Task Force Présidentielle ».

Tous ces instruments juridiques ont été pris sur fond de la Constitution. Ils tirent leur validité de la Constitution en vigueur qui prévoit les droit de l'homme notamment les droits économiques, sociaux et culturels ${ }^{14}$. En ce qui concerne la limitation des droits de manière générale, cette Constitution interdit toute limitation des droits de l'homme qui literait le droit à la vie; l'interdiction de la torture et des peines ou traitements cruels, inhumains ou dégradants; l'interdiction de l'esclavage et de la servitude; le principe de la légalité des infractions et des peines; les droits de la défense et le droit de recours; l'interdiction de l'emprisonnement pour dettes ou la liberté de pensée, de conscience et de religion.

L'Ordonnance du Président de la République qui a proclamé l'état d'urgence sanitaire a limité plusieurs droits de l'homme en RDC. L'arrêté du Vice-premier Ministre, ministre de l'intérieur, sécurité et affaire coutumier n ${ }^{\circ}$ 25/CAB/VPM/MININTERSECAC/GKM/06/20 20 du 25 mars 2020 portant modalités pratiques d'exécution des mesures de limitation de la propagation de la pandémie de Covid-19 tout en arrêtant des mesures d'application de l'ordonnance du Président de la république, celui-ci est venu durcir les limitations des droits de l'homme pendant la période de l'état d'urgence dû au Covid-19. Ces limitations des droits de l'homme ont touché la quasi-totalité des droits dont jouit la personne humaine en RDC. Avant d'insister sur les droits économiques, sociaux et culturels qui ont été visés, nous allons présenter ici tout d'abord une vue générale de la situation des autres droits qui ont été touché par les mesures de l'état d'urgence en RDC.

La Constitution de la RDC en vigueur garantie à toute personne qui se trouve sur le territoire national le droit d'y circuler librement, d'y fixer sa résidence, de le quitter et d'y revenir. Mais suite à la proclamation de l'état d'urgence sanitaire, il a été fait application de la

14 Lire le Titre II, Chapitre 2 de la Constitution de la RDC du 18 février 2006 telle que modifiée en 2011. 
fermeture de toutes les frontières du pays aux passagers et à toute personne, sauf pour les navires cargos et autres moyens de transport frets qui sont autorisés à accéder au territoire national. Mêmes les citoyens congolais se trouvant à l'extérieurs, et surtout ceux se trouvant dans les pays à risque voire même dans les pays de transits, en principe ils ne pouvaient pas accéder à l'intérieur du territoire nationale. Pour ceux qui se trouvaient à l'intérieur du territoire national pendant ce temps, la situation n'était pas du tout facile pour eux aussi, puisque il était interdit tous les voyages de la capitale vers les Provinces et vice-versa. Et plus tard, ce fut le confinement des populations dans leurs domiciles et même quand il fallait mettre fin au confinement de la population dans leurs domiciles, le pays a été frappé par le couvre-feu général qui exigeait à la population à prendre des précautions pour retourner à leurs domiciles au plus tard à $21 \mathrm{~h} 00$. Ainsi, la liberté de circuler en RDC a été frappé des plusieurs formes de limitation.

Il a également était interdit tous rassemblements, réunions et célébrations sur les voies et lieux publics en dehors du domicile familiale ${ }^{15}$. Mais la Constitution en vigueur garantie la liberté des réunions pacifiques et sans armes sous réserve du respect de la loi, de l'ordre public et des bonnes mœurs ${ }^{16}$ mais aussi la liberté de manifestation est garantie tout en imposant aux organisateurs simplement d'informer par écrit l'autorité administrative compétente ${ }^{17}$.

L'ordonnance proclamant l'état d'urgence sanitaire à fermer provisoirement sur toute l'étendue du territoire national les écoles, les universités, les Instituts supérieurs officiels et privés et tous établissement recevant du public ainsi que des lieux de réunion voire même la suspension des tous les cultes religieux ainsi que toutes les activités sportives qui attirent beaucoup des personnes ${ }^{18}$. La Constitution en vigueur en imposant au pouvoir publics l'obligation de protéger la jeunesse contre notamment toute atteinte à son éducation et son développement intégral, ceux-ci, les pouvoirs publics, devraient plutôt se battre contre la propagation du virus de Covid-19, en prenant des dispositions pour que la jeunesse puisse aller à l'école sans risque d'être contaminer. Telle serait se conformer à son obligation constitutionnelle découlant des articles 42 et 43 puisque cette situation de fermeture des établissements a perduré pendant plusieurs au mépris des critiques acerbes de la population qui fustigeait l'irrationalité de cette mesure allant jusqu'aux Provinces qui n'ont pas été dangereusement secouées par la propagation du virus Covid-19.

Les droits économiques, sociaux et culturels qui ont été le plus limités par les mesures de l'état d'urgence sanitaire en RDC sont plus les droits liés à la mobilité humaine. Ainsi, par manque des infrastructures adéquates, le confinement de la population à leurs domiciles suivit du couvre-feu général, ont eu un impact remarquable sur le droit au travail sous plusieurs aspects. Certains citoyens ont été retrouvés dans l'impossibilité d'aller à leurs lieux

15 Article 3 de l'Ordonnance du Président de la République portant état d'urgence.

16 Article 25 de la Constitution de la RDC du 18 février 2006.

17 Article 26 de la Constitution de la RDC du 18 février 2006.

18 Article 3 de l'Ordonnance du Président de la République proclamant l'état d'urgence sanitaire. 
de travail ou d'exercer leur travail, d'autres encore ont été victimes des suppressions massives de leurs emplois à cause de la crise financière engendrée par la pandémie de Covid-19.

D'autres citoyens encore ont été simplement interdits d'exercer leurs activités qui constituent leur travail par les mesures qui ont proclamé et régie la période de l'état d'urgence sanitaire en RDC. Dans cette catégorie il est retrouvé notamment les travailleurs des discothèques, bars, cafés, terrasses et restaurants voire même les petits commerces et le commerce informels qui sont des activités qui occupent une grande partie de la population congolaise. Certaines de ces limitations des droits de l'homme sont allés au-delà même de la durée de l'état d'urgence en dépit du fait que la Constitution congolaise en vigueur qualifie certains de ces droits comme des droits sacrés.

\section{L'Application des mesures de limitation des droits de l'homme par l'Etat d'urgence}

Plusieurs institutions ont été sollicitées pour intervenir dans l'application des mesures éditées pour la période de l'état d'urgence sanitaire. Il faut noter qu'aux côtés des institutions existantes, plusieurs autres institutions « spécialisées » ont vu le jour pour apporter un soutien spécial à la situation de la propagation de la pandémie de Covid-19 sur le territoire national.

Les responsables et chefs des institutions du Pouvoir central, des provinces ainsi que des Entités territoriales décentralisées et la cellule de riposte devraient veiller, chacun en ce qui le concerne, à l'exécution de l'Ordonnance du Président de la République qui a proclamé l'état d'urgence sanitaire en RDC. Les services qui étaient au premier rang sont notamment les services de sécurité et d'ordre public; ceux de la santé publique et ceux de l'économie nationale.

En ce qui concerne la sécurité public pendant la période de l'état d'urgence, le problème de respect et de protection des droits de l'homme a été posé de manière très interpellative à l'égard des autorités publiques mais aussi, à l'égard des défenseurs des droits de l'homme. Le Ministre de l'intérieur et e la sécurité a créé par Arrêté ministériel un Comité de suivi et de l'évaluation de la mise en œuvre des mesures de la riposte contre le Covid-19 au niveau de chaque province, Ville, Commune, territoire et secteur. Ce comité devrait être composé des membres habituels des Comités locaux de sécurité élargi aux représentants des différents ministères et services sectoriels impliqué dans la riposte contre la pandémie de Covid-19 en RDC.

Les membres habituels des Comités locaux de sécurité dont il est question ici sont les autorités et agents de la police administrative, la police militaire et autres agences de sécurité comme la politique criminelles ou les agences de renseignement nationales. Ceci, livré à eux-mêmes au milieu de la population, ils se sont retrouvés comme maitre et roi sur le terrain à telle enseigne que, au vue de la situation générale des différents corps de défense et de sécurité nationale, les policiers et les militaires déployés sur le terrain se sont caractérisés par plusieurs bavures et violations des droits de l'homme tantôt en Monnayant l'inob- 
servances des certaines mesures ou en outrepassant leurs compétences bien qu'exceptionnelles pour la situation qui prévalait au pays.

En ce qui concerne les responsable de santé publique auxquelles le pouvoir public a fait appel pour être au-devant de la scène pendant cette période de l'état d'urgence sanitaire, les dangers et les risques liés à la limitation des droits de l'homme étaient encore plus complexe. Les raisons sont multiples, mais trois d'entre elles peuvent être soulevés ici. D'abord, en RDC ces personnels de santé, médecins généralistes ou spécialistes, et infirmiers et les paramédicaux, veuillent moins aux questions relatives aux droits de l'homme. Il suffit de jeter un coup d'œil sur la façon dont ils posent leurs actes médicaux pour s'en rendre compte : la loi et leur serment leurs obligent de veiller d'abord à la santé des patients avant les questions financières, mais c'est tout l'inverse dans leur pratique en RDC; la loi leur demande de tenir informer le patient ou un membre de famille proche de tous les actes médicaux qu'ils envisagent poser sur eux, mais c'est à peine ou exceptionnellement que cela est faite.

Ensuite, pendant cette période de l'état d'urgence, le pouvoir public a mis entre les mains des personnels de santé des pouvoirs exorbitants. En matière des droits de l'homme, le célèbre docteur Muyembe avait le pouvoir de «confiner » ou de « déconfiner » et cela était expliqué de manière anecdotique dans la capitale kinoise en disant « Muyembe ko confiner biso te » c'est-à-dire Muyembe ne nous confinez pas! Concrètement, par ses recommandations au Président de la République, il avait le pouvoir de limiter le droit de la population de circuler librement sur le territoire nationale, d'y entrer ou d'y sortir, mais aussi le pouvoir d'empêcher les citoyens d'aller à leur lieux de travail, de manifester même pacifiquement, de recevoir une éducation puisqu'il avait le pouvoir de recommander la fermeture des écoles, le droit de culte et des croyances puisqu'il pouvait même recommander la fermeture des églises. A ce niveau, le danger était que le Docteur et ses services répondaient directement du Chef de l'Etat par sa Cellule de riposte qui était directement attaché à la Présidence de la République. Les représentant du peuples, voire même le pouvoir judicaire bien qu'étant actifs, eux aussi étaient comme mis à l'écart à cause de la peur de la propagation de la pandémie. Il suffit de jeter un coup d'œil sur la forme et la procédure exigé par la Constitution en vigueur en ce qui concerne la proclamation de l'état d'urgence comparativement à la manière dont la première ordonnance du Président de la république à été prise, suivit des procédures de régularisation par l'Assemblée Nationale et le Sénat.

En fin, plusieurs nouvelles institutions ont été créées pour le besoin de la cause, mais certaines d'entre elles tombaient quasiment dans une sorte de dédoublement ou inflation institutionnelles. Il s'agit de la Cellule de riposte contre le Covid 19 en RDC; la Task force présidentielle; le Secrétariat technique; le Comité multisectoriel de riposte (CMR-COVID-19); voire même Le Fonds national de solidarité contre le Coronavirus. 


\section{Conclusion}

Les droits de l'homme s'arrêtent-ils le jour de la proclamation de l'état d'urgence? cet article dédié à la problématique de la limitation des droits économiques, sociaux et culturels en RDC pendant la période de l'état d'urgence sanitaire à fait un tableau succincte de l'état de lieux des droits de l'hommes pendant la période de l'état d'urgence sanitaire en RDC avec un regard sur les droits économiques, sociaux et culturels, les droits de la deuxième génération, dont parfois la justiciabilité reste sujette à discutions et appréciations.

Les mesures proclamant l'état d'urgence ont limités plusieurs droits de l'homme en RDC. Et les dans cet article il a été relevé que les limitations explicites de certains droit entrainaient indirectement la limitation des autres droit de l'homme. Notamment, quand le droit de circuler librement sur le territoire national est limité, celle touche le droit au travail, le droit à l'éducation voire même le droit au culte et entrave le droit d'exercer sa croyance. Et le droit d'entrer et de sortir du territoire national n'a pas été épargné par la proclamation de l'état d'urgence.

Pendant une période où tout le monde craignait pour sa santé, les animateurs des pouvoirs publics n'ont pas fait exception. Et cette situation n'a pas laissé sans crainte le protection et la défense des droits de l'homme. Les personnels de santé se sont vus octroyés des prérogatives exceptionnelles, pour le moins, dangereux pour la promotion et la protection des droits de l'homme et d'autres valeurs et principes de l'Etat de droit.

\section{Bibliographie}

\section{Textes juridiques}

Arrêté ministériel No. 25/CAB/VPM/ MININTERSECAC/GKM/06/2020 du 25 mars 2020 portant modalités pratiques d'exécution des mesures de limitation de la propagation de la pandémie de Covid-19

Constitution de la RDC du 18 février 2006, in J.O.R.D.C, 47ème année, numéro spécial du 18 février 2006 telle que modifiée en 2011.

Ordonnance n²0/029 du 23 avril 2020 portant mesures complémentaires nécessaires pour faire face à l'épidémie au Covid 19.

Ordonnance n`20/033 du 02 mai 2020 modifiant et complétant l'ordonnance n²0/018 du 06 avril 2020 portant création, organisation et fonctionnement d'un Fonds national de solidarité contre le Coronavirus, en sigle « FNSCC »

Ordonnance n ${ }^{\circ} 20 / 036$ du 02 mai 2020 portant création, organisation et fonctionnement d'une commission ad hoc dénommée « Task Force Présidentielle».

Ordonnance n ${ }^{\circ} 20-018$ du 6 avril 2020 portant création, organisation et fonctionnement d'un Fonds national de solidarité contre le Coronavirus, en sigle « FNSCC

Ordonnance numéro 20/014 du 24 mars 2020 portant proclamation de l'état d'urgence sanitaire pour faire face à la pandémie de Covid-19 
Pacte international relatif aux droits économiques, sociaux et culturels («PIDESC ») du 16 décembre 1966

\section{Ouvrages}

BOUTROS BOUTROS GHALI, L'Organisation de l'unité africaine : bilan et perspectives, Kinshasa, 1986.

COLLARD D., " La Déclaration universelle des droits de l'homme a quarante ans », in Défense nationale, problèmes politiques, économiques, scientifiques militaires, $45^{\text {ème }}$ année, janvier 1989.

COMBACAU J. et SUR S., Droit international public, Paris, 2006.

FRANS VILJOEN \& CHIDI ODINKALU, La prohibition de la torture des mauvais traitements dans le système africain des droits de l'homme : guide pratique juridique à l'intention des victimes et de leurs défenseurs, Genève, OMCT, Collection de guides juridiques de l'OMCT, vol. 3, 2006.

LEAH LEVIN., Droits de l'homme : questions et réponses, Paris, Editions de l'Unesco, nouvelle édition revue, augmentée et mise à jour, 1997.

LUDOVIC HENNEBEL et HÉLÈNE TIGROUDJA, Traité de droit international des droits de l'homme, Paris, 2016

MBATA BETUKUMESU MANGU A., Abolition de la peine de mort et constitutionnalisme en Afrique, Paris, 2011

MOHAMED BEDJAOUI, Droit international : bilan et perspectives, Tome 2, Paris, Editions A. Pedone et Unesco, 1991.

NTUMBA LUABA LUMU, « La Charte africaine des droits de l'homme et des peuples », in ZaïreAfrique $\mathrm{n}^{\circ} 164,1982$.

TSHIBANGU KALALA, Code des organisations internationales, Bruxelles, 2008,

WACHSMANN P., Les droits de l'homme, Paris, 2002.

\section{Articles}

GREWE C., «L'avenir de la Cour européenne des droits de l'homme : réflexions à propos du colloque de Graz des 7 et 8 février 2003 ", in Revue universelle des droits de l'homme, Vol. 15, n 7-10, 2003, p. 249.

Mireille Delmas-Marty, " Le crime contre l'humanité, les droits de l'homme et l'irréductible humain », Revue de science criminelle et de droit pénal comparé, 1994, n 3, p. 477- 490

\section{Rapports et autres documents}

COMMISSION AFRICAINE DES DROITS DE L'HOMME ET DES PEUPLES, $32^{\text {ème }}$ et $33^{\text {ème }}$ rapports d'activités combinés de la Commission africaine des droits de l'homme et des peuples présenté conformément à l'article 54 de la Charte africaine des droits de l'homme et des peuples, disponible en ligne à l'adresse http://www.achpr.org/files/activity-reports/32-and-33/achpr5152_actre p32and33_fr.pdf(21 mai 2013). 
COMMISSION AFRICAINE DES DROITS DE L'HOMME ET DES PEUPLES, Documentation, $\mathrm{n}^{\circ}$ 1 , Banjul.

.Haut-Commissariat des Nations Unies aux droits de l'homme, « Emergency measures and covid-19

INSTITUT POUR LES DROITS HUMAINS ET LE DEVELOPPEMENT EN AFRIQUE, Compilations des décisions sur les communications de la Commission africaine des droits de l'homme et des peuples, extraits des rapports d'activités, 1994-2001, Dakar, Institut pour les droits humains et le développement en Afrique, 2002

INSTITUTE FOR HUMAN RIGHTS AND DEVELOPMENT IN AFRICA, Décisions de la Commission africaine des droits de l'homme et des peuples sur les Communications, 2002-2007, Cape Town, Institue for Human Rights and Development in Africa, 2008. 